Obere Extremität $2021 \cdot 16: 247-254$ https://doi.org/10.1007/s11678-021-00657-6 Received: 3 May 2021 Accepted: 26 May 2021

Published online: 3 September 2021

(c) The Author(s) 2021

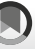

\section{Joint-preserving surgical treatment options for irreparable posterosuperior rotator cuff tear}

\author{
Partial repair, superior capsular reconstruction, latissimus \\ dorsi, lower trapezius, or a balloon?
}

\author{
Florian Grubhofer ${ }^{1}$ (D) · Jon JP Warner ${ }^{2}$ \\ ' University Hospital Balgrist, University of Zurich, Zurich, Switzerland \\ ${ }^{2}$ Harvard Shoulder Service, Massachusetts General Hospital, Harvard Medical School, Boston, USA
}

\title{
Abstract
}

Treatment of irreparable rotator cuff tears in young active patients is challenging. A variety of therapeutic options are available. Only a few joint-preserving treatment options show reliable improvements over a long-term follow-up period. However, the treatment outcomes of joint preservation procedures are not comparable to those of RTSA, as patients are typically younger and have higher expectations. It is remarkable that most of the joint-preserving therapeutic options for irreparable rotator cuff ruptures lack long-term treatment results. This article highlights the indications, technical aspects, and treatment outcomes of the most commonly performed jointpreserving surgeries for irreparable rotator cuff rupture.

\section{Keywords}

Biomechanical phenomena · Arthroplasty, replacement, shoulder · Tendon transfer · Shoulder prosthesis $\cdot$ Tenodesis

\section{Introduction}

The authors strongly believe that the success of treatment of irreparable posterosuperior rotator cuff tear (IPRCT) depends primarily on the indication for the respective treatment, and secondarily on the correct technical execution of the operation per se.

Different joint-preserving treatment options exist, but an established therapeutic algorithm does not yet exist even though shoulder societies like the American Shoulder and Elbow Surgeons (ASES) are actively striving for it. For example, the Neer Circle (elected association of 110 ASES members) tried to establish a treatment algorithm based on expert opinion using a Delphi process. It was found that there was consensus for only a few non-joint-preserving scenarios - the majority of the experts' approaches dif- fered, particularly in the joint-preserving scenarios.

The joint-preserving surgical treatment methods discussed in this article include:

- arthroscopic partial repair (APR) + biceps tenodesis or tenotomy

- superior capsular reconstruction (SCR)

- tendon transfer (lower trapezius and latissimus dorsi)

- subacromial balloon implantation

The treatment decision-making process depends, from the authors' point of view, on the three following factors:

1. biological age

2. chief complaint (pain, weakness, stiffness)

3. rotator cuff tear (RCT) pattern according to the classification of Collin et al. [7]; • Fig. 1

In this review, joint-preserving surgical treatment options are highlighted in terms 


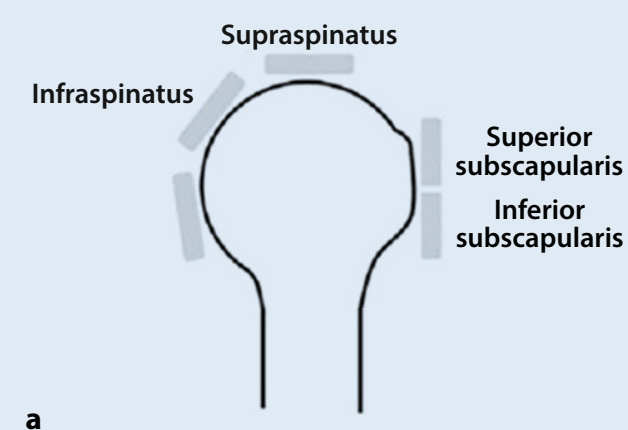

a
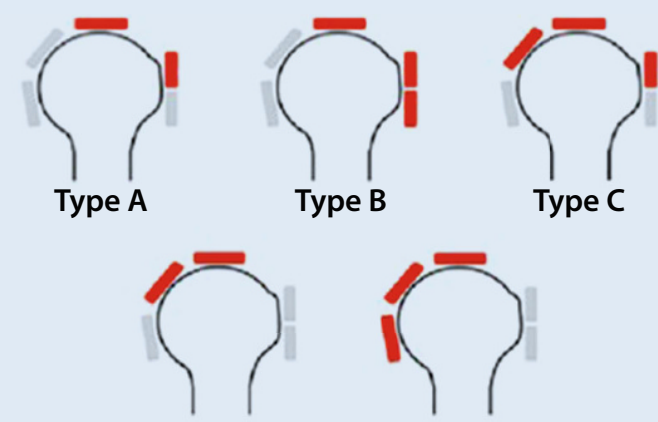

Type D

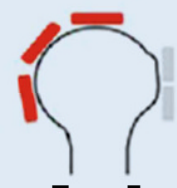

Type E
Fig. $1<$ Descriptive classification of irreparable posterosuperior rotator cuff tears according to Collin et al. [7]. (a) Schematic lateral view on humerus with anterior Subscapularis tendon, superior Supraspinatus tendon, posterior Infraspinatus and Teres minor tendon, (b) Schematic illustration of irreparable tendon tears (illustrated in red) of biomechanics, surgical technique, and treatment outcomes. In addition, a treatment algorithm used in the authors' clinics is presented (- Fig. 2).

\section{Arthroscopic partial repair + biceps tenodesis/tenotomy}

\section{Indication according to the treatment algorithm}

- Biological age: young patients with low demands

- Chief complaint: pain, pain combined with stiffness

- Pattern of irreparable RCT: A, C, D, E

\section{Strengths and weaknesses}

- + All-arthroscopic procedure, promising mid-term outcomes

- - Weakness for external rotation or flexion is better addressed with a tendon transfer. Long-term data lacking

\section{Abbreviations}

APR Arthroscopic partial repair

ASES American Shoulder and Elbow Surgeons

IPRCT Irreparable posterosuperior rotator cuff tear

JSES Journal of Shoulder and Elbow Surgery

LDT Latissimus dorsi tendon transfer

LTT Lower trapezius transfer

RTSA Reverse total shoulder arthroplasty

$S C R \quad$ Suprascapular reconstruction

\section{Biomechanical considerations and} surgical technique

The goal of arthroscopic partial repair is to restore the force couple consisting of the remaining subscapularis and infraspinatus tendons [5]. Complete anatomic reconstruction of the rotator cuff is no longer possible because of the advanced retraction. The upper edge of the infraspinatus tendon is reattached as far as possible to the original insertion site. The biceps tendon is either tenodesized or tenodized. It is known and has been published by Boileau et al. that treatment of the biceps alone significantly improves shoulder function and satisfaction in patients with massive irreparable RCTs [3]. In patients with irreparable posterosuperior rotator cuff tears (IPRCT) and concomitant shoulder stiffness, the treatment results of tendon transfer are poor; accordingly, from the authors' point of view, shoulder stiffness is a relative contraindication for tendon transfers or SCR. In young patients with a painful and at the same time stiffened shoulder, APR can be considered as a treatment option and should be combined with arthroscopic capsular release. Postoperative mobilization begins passively from the first postoperative day, with actively assisted range-ofmotion exercises from the 4th-6th postoperative week. Gentle strengthening to a maximum of $5 \mathrm{~kg}$ begins between the 10 th and 12th postoperative weeks. Full weightbearing is allowed from the 16th postoperative week.

\section{Results}

The clinical results of APR in short- and mid-term follow-up show significantly improved shoulder function and patient satisfaction [8, 20, 27, 33]. Interestingly, the 5 -year outcomes of patients treated with APR for irreparable massive rotator cuff tear are comparable to the outcomes of patients who underwent anatomic arthroscopic reconstruction of reparable massive RCTs [21]. The integrity of the teres minor was found to be an important predictive factor $[1,29]$. Radiologically, re-rupture of the partial repair was described in $49 \%$ [23]. No clinical outcome differences were seen between patients after APR versus arthroscopically assisted latissimus dorsi tendon transfer (LDT) during a short-term follow-up period [1]. A randomized trial demonstrated no significant difference in clinical treatment outcomes 2 years after APR vs. SCR in 41 patients [19].

\section{Superior capsular reconstruction}

\section{Indication according to the} algorithm

- Biological age: young patients with low demands.

- Chief complaint: pain

- Pattern of irreparable RCT: A, isolated irreparable supraspinatus tear

\section{Strengths and weaknesses}

- + All-arthroscopic procedure (if allograft is used).

- - Allo-/or autograft (fascia lata) necessary, long-term outcome data pending, 


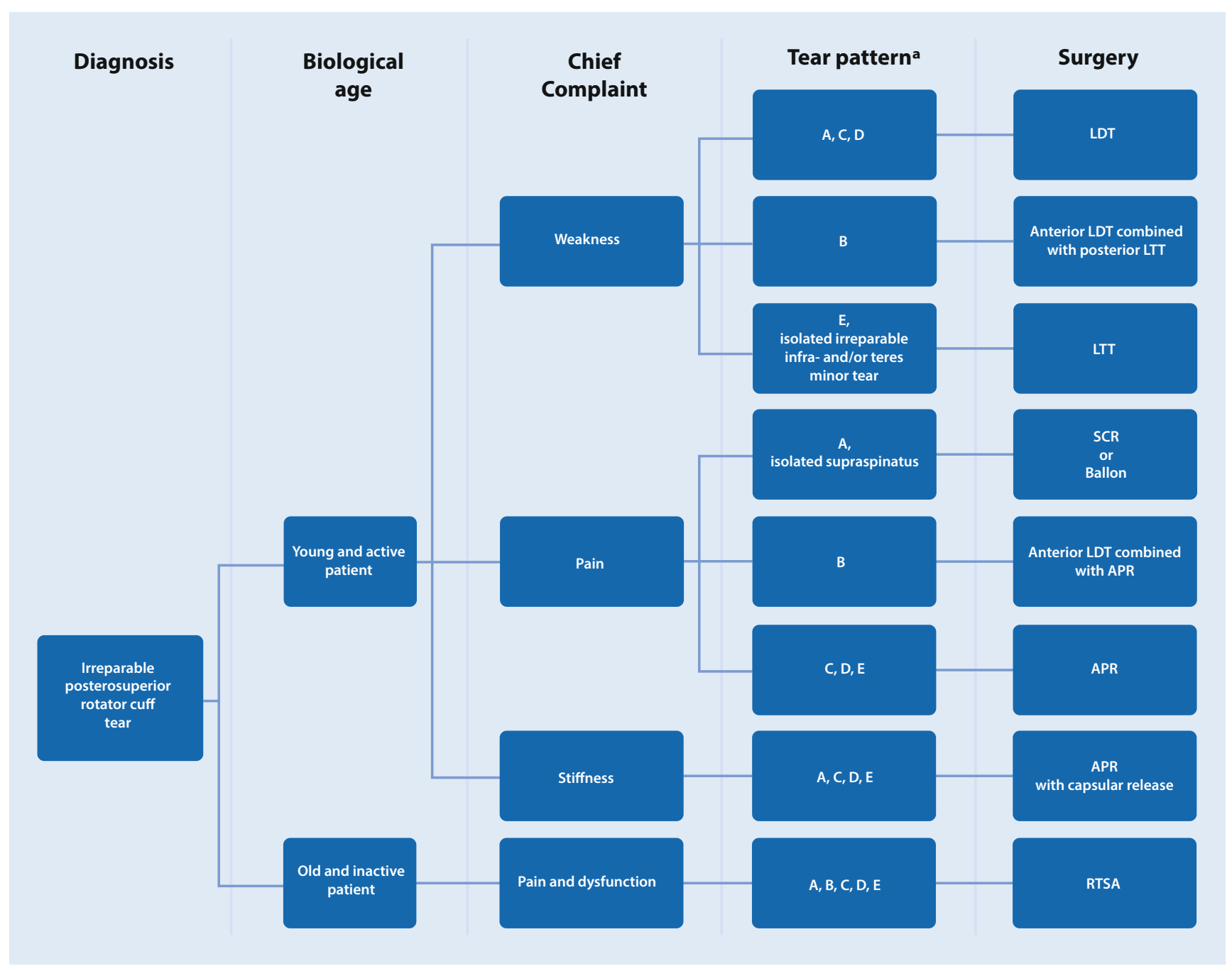

Fig. $2 \Delta$ Flowchart of the authors' preferred treatment algorithm of irreparable posterosuperior rotator cuff. LDT latissimus dorsi tendon transfer, LTT lower trapezius transfer, SCR suprascapular reconstruction, APR arthroscopic partial repair, RTSA reverse total shoulder arthroplasty. ${ }^{a}$ according to Collin Classification for irreparable rotator cuff tears

implant costs (allograft and multiple anchors).

- Risk factors for poor outcome: irreparable posterior rotator cuff rupture (infraspinatus and/or especially with involvement of teres minor), surgeon's experience $(<10)$.

\section{Biomechanics}

According to SCR inventor T. Mihata, implantation of an auto- or allograft membrane that replaces the superior capsule and the irreparable rotator cuff leads to improved vertical stability and thus reduces subacromial impingement [25]. Furthermore, the implanted membrane acts as an anchor for the remaining subscapularis and infraspinatus tendons, leading to an increase in their tendon tension and thereby restoring the force couple [26].

\section{Surgical technique}

The procedure is performed arthroscopically in a beach chair position. At the authors' institutions, a 3-mm dermal allograft is used. After thorough subacromial debridement and arthroscopic biceps tenotomy or tenodesis, partial repair of the remaining rotator cuff is performed if possible. The superior glenoid is debrided and exposed to fix the allograft with two to three anchors medially between the 10 and 12 o'clock positions. Fixation on the lateral aspect of the greater tuberosity is achieved with a double-row suture bridge technique with the arm held at $45^{\circ}$ of abduction.
Postoperatively, patients are immobilized in a 45-degree abduction pillow for a total of 6 weeks, starting with passive range of motion between 4 and 6 weeks and active assistive range of motion between 6 and 8 weeks. Light weightbearing is allowed after 10 to 12 weeks. Unlimited weight bearing is allowed 16 weeks postoperatively.

\section{Results}

Clinical outcomes from the authors' clinic (Massachusetts General Hospital) were published in the Journal of Shoulder and Elbow Surgery in 2019 [34]. The results were exceedingly modest. In total, SCR surgery was performed in 65 patients by 6 different fellowship-trained shoulder 
surgeons within the period from January 2015 to November 2017; however, 31 patients had to be excluded as they were not available for 12-month followup. Of the 34 patients included, $65 \%$ were dissatisfied with the treatment outcome at a mean follow-up of 12 months. The 1- and 2-year failure-free survival rates were 34 and $16 \%$, respectively. The 1 - and 2-year "free of reoperation rate" was 64 and 44\%, respectively. There was no significant improvement in shoulder function or pain with SCR surgery. Risk factors for a poor treatment outcome were the extent of fatty infiltration in the infraspinatus muscles and the number of SCR operations performed by the operating surgeon $(<10)$. It should be emphasized that numerous level III and IV studies exist that show good to excellent short- and mid-term results, even in patients with preoperative pseudoparalysis $[4,6,24]$.

Nevertheless, the poor treatment results at the authors' institution (Massachusetts General Hospital) have led to a drastic reduction of the indication for $\mathrm{SCR}$, which is now only considered in patients with strictly isolated supraspinatus tendon tears or type A lesions. The majority of patients-especially those with advanced fatty degeneration of the posterosuperior rotator cuff muscles (infraspinatus and teres minor)-are treated according to the treatment algorithm with tendon transfer.

\section{Lower trapezius transfer}

Indication according to the algorithm

- Biological age: young patients with high demands

- Chief complaint: weakness; weakness for external rotation > weakness for flexion

- Pattern of irreparable RCT: A, C, D, E or isolated irreparable infraspinatus and teres minor tear.

\section{Strengths and weaknesses}

- + Addresses posterior cuff deficiency of infraspinatus AND teres minor, technically less demanding than latissimus dorsi transfer-especially in obese patients, force vector of the lower trapezius similar to posterior cuff muscles, primarily synergistic muscle (lower trapezius muscle contracts with external rotation). Potential treatment option in patients with an additional irreparable subscapularis lesion (with combined anterior latissimus dorsi transfer; Elhassan B.; in submission process, not yet published).

- - Auto- or allograft necessary, longterm outcome data pending, partially open procedure, implant cost (allograft).

- Risk factors for poor outcome: diabetes mellitus $\rightarrow$ stiffness, deltoid dysfunction, non-compliance.

\section{Biomechanics}

LTT was originally developed to treat patients with brachial plexus palsy $[2,11]$. These patients suffer in particular from a lack of external rotation due to failure of the external rotator muscles (infraspinatus and teres minor). In addition, cervical nerve root 5 is frequently involved, which causes dysfunction of the deltoid muscle. Prior to development of LTT, these patients with deltoid dysfunction were treated with LDT. A common complication in these patients was posterior (sub)dislocation, which led to the search for an alternative tendon transfer to the LDT $[2,11]$. In this regard, LTT was developed by Elhassan to address these patients with less complications [13]. It was shown biomechanically that LTT transfer, leaving the latissimus dorsi tendon intact, did not present posterior instability problems in these patients with deltoid dysfunction [12].

Subsequently, this technique was also used in patients with irreparable rotator cuff rupture. Biomechanically, the force vector of the inferior trapezius portion was found to be similar to that of the infraspinatus and teres minor muscles. Another advantage of the lower trapezius muscle is its synergistic action with the infraspinatus and teres minor, as the muscle contracts during glenohumeral external rotation. A disadvantage of the lower trapezius muscle is the reduced excursion of the muscle as well as the short tendon length, which makes direct fixation to the greater tuberosity impossible. Therefore, interpositional tenodesis with an allograft or autograft is necessary.

\section{Surgical technique}

The technique is described very clearly in the publication by Wagner et al. [14]. Here, the authors wish to point out the most important steps and possible sources of error, which are crucial for the outcome of the operation in their opinion.

Positioning. The procedure can be performed in a beach chair or lateral decubitus position. The positioning is crucial for this surgery. Positioning and subsequent draping must allow for good exposure of the medial scapular border. A common mistake is that the scapula is insufficiently exposed.

Skin incision. Originally, an L-shaped skin incision was described. Nowadays, an oblique skin incision which starts medially $1 \mathrm{~cm}$ medial to the scapular rim and $1 \mathrm{~cm}$ inferior to the spina scapulae and is guided $4-5 \mathrm{~cm}$ laterally, parallel to the course of the scapula spine, is preferred. A common source of error here is that the skin incision is placed too far laterally. This can lead to difficult exposure of the inferior trapezius muscle-tendon unit.

Lower trapezius exposure. Removal of the fat tissue pad that covers the fascia of the lower trapezius muscle and tendon makes exposure of the lower trapezius easier. At the lateral margin of the lower trapezius, the layer between the trapezius tendon and infraspinatus fascia can be developed by blunt dissection with the finger. If this layer can be developed, harvest of the lower trapezius tendon is easy to perform.

Preparation of the graft channel. Tension- and kinking-free sliding for the auto/ allograft is mandatory. Often the muscle fascia of the infraspinatus muscle causes mechanical restrictions for the graft, and it is therefore important that it be sufficiently released. 
Arm position during graft-trapezius tendon tenodesis. During tenodesis the arm is placed in combined maximal external rotation and $60-90^{\circ}$ of abduction. After tenodesis and postoperatively, it is crucial that the arm be kept in maximum external rotation and immobilized in an external rotation brace. The tension created on the back during internal rotation of the arm is very high and could endanger the tenodesis. Accordingly, it is also very important to assume a high level of brace-wearing compliance.

\section{Results}

In the study by Elhassan et al., a significant improvement in function, pain, and subjective shoulder score was observed in 32 of 33 patients (mean age 53 years) after a mean follow-up duration of 47 months [13]. The better the active and passive mobility preoperatively, the more likely a good treatment outcome can be assumed. Accordingly, from the authors' point of view, shoulder stiffness is a (relative) contraindication for tendon transfers. Another study by Elhassan demonstrated significant improvement in shoulder function and patient satisfaction 12 months postoperatively in $90 \%$ of a total of 41 patients [14]. True pseudoparalysis was present in $46 \%$ (19 patients) preoperatively. In 18 of the 19 pseudoparalytic patients, the pseudoparalysis resolved and a significant improvement in shoulder function and patient satisfaction was achieved.

\section{Latissimus dorsi transfer}

Indication according to the
algorithm

- Biological age: young patients with high demands

- Chief complaint: weakness; weakness for flexion > weakness for external rotation

- Pattern of irreparable RCT: A, C, D

\section{Strengths and weaknesses}

- + Long-term data available showing sustained improvement even after 12 years. Great excursion of the latissimus muscle, no graft needed, low implant costs (2-3 anchors).

- - Technically challenging, treatment outcome dependent on the integrity of the teres minor and subscapularis muscle, partially open procedure, latissimus function out of phase (internal rotator, vertical force vector).

- Risk factors for poor outcome: shoulder stiffness, irreparable subscapularis tear, teres minor atrophy, pseudoparalysis, high critical shoulder angle, previous rotator cuff surgeries.

\section{Biomechanics}

The pioneering description of LDT for chronic posterosuperior RCTs was presented by Gerber in 1988 [17].

The latissimus dorsi acts as a powerful internal rotator, adductor, and extensor of the humerus in the glenohumeral joint. The force vector is significantly more vertical than the lower trapezius portion due to its natural course. However, the latissimus dorsi muscle exhibits such good excursion that the tendon can be inserted directly at the superolateral facet of the greater tuberosity. The transfer restores the force couple of the rotator cuff, allowing centering of the humeral head during deltoid activation.

\section{Surgical technique}

The procedure was first described as an open procedure in a lateral decubitus position [15]. Nowadays, the majority of the procedure is arthroscopically assisted, with the patient in the beach chair position and the tendon being harvested openly through a skin incision along the posterior axillary fold [18]. The all-arthroscopic technique has also been described, although it has not yet become widely performed [9]. The advantages of the arthroscopically assisted approach are the ability to supply the subscapularis tendon, perform a partial repair of the posterosuperior rotator cuff tendons if possible, sparing of the deltoid muscle, and less scarring.

There are several descriptions of arthroscopically assisted LDT. A particularly illustrative presentation of the single surgical steps can be viewed on VuMedi. link: https://www.vumedi. com/share/a5eb4a01-32d6-4aac-a3003b099041e440/.

Herein, the authors focus on the pearls and pitfalls of the technique:

Positioning. In beach chair positioning, an arm holder can be attached ipsilaterally. With maximum flexion and internal rotation of the arm, the posterior axial fold, which corresponds to the latissimus dorsi muscle and tendon, is stretched to the maximum. In this position, the skin incision and harvest of the tendon from the humerus should be performed.

Harvest. The latissimus tendon is wide and long, and the teres major tendon is short. The layer between the latissimus tendon and muscle belly must be clearly separated before tendon release. Tendon release can be performed either openly via the axilla or arthroscopically.

Muscular release. The latissimus muscle has a very high excursion if the muscular release is performed adequately. The muscle belly should be released posteriorly and far distally. Anteriorly, attention must be paid to the nerve pedicle, which enters the muscle anteriorly about $14 \mathrm{~cm}$ distal to the tendinous attachment.

Tendon shuttle. The layer between the deltoid muscle and the posterior cuff muscles (infraspinatus and teres minor) should be dissected wide open, both from intraarticularly and via the open approach posteriorly to allow the tendon transfer to glide freely.

Fixation of the tendon. The anteromedial and anterolateral tagging sutures are fixed anteriorly at the superolateral aspect of the greater tuberosity via knotless anchors. The fixation is facilitated if the respective sutures are shuttled out through a separate anteromedial and anterolateral portal. A third anchor might be needed at the posterior aspect or the greater tuberosity to avoid windshield whipper effect of the tendon.

Postoperative rehabilitation. Patient compliance is important and should be assessed preoperatively. Postoperative immobilization in a 30-degree abduction 
pad for 6-8 weeks. Passive and active assistive exercise therapy after 8 weeks, gentle strengthening after 12 weeks, and transition to full weightbearing after 16 weeks.

Results

A major strength of LDT as a treatment option is that it is the only therapeutic option for the treatment of IPRCT for which long-term results are available $[10,16]$. In the study by Gerber et al., with a mean follow-up of 12 years (minimum follow-up of 10 years), it was shown that shoulder function (Constant score \% pre-vs. postoperative: 56 vs $80 \%$ ) and patient satisfaction (subjective shoulder value from preoperative 29 to postoperative $70 \%$ ) of 44 patients with 46 operated shoulders were sustainably improved over this long period [16]. Mid-term results of the newer arthroscopically assisted technique are also available and do not differ significantly from the open technique [32]. Long-term failure rates range from $10 \%$ [10] to $14 \%$ [32] and $30 \%$ [16].

Over the years, the following risk factors for poor outcome have been identified: fatty atrophy of the teres minor muscle, preoperative pseudoparalysis, excessively high critical shoulder angle, irreparable subscapularis tear, shoulder stiffness, and previous rotator cuff procedures.

\section{Subacromial balloon}

\section{Indication according to the algorithm}

- Biological age: young patients with low demands

- Chief complaint: pain

- Pattern of irreparable RCT: A, isolated irreparable supraspinatus tear

\section{Strengths and weaknesses}

- + Short operation time, easy to implement surgically, all-arthroscopic procedure.

- - Not US Food and Drug Administration (FDA) approved, no long-term data available, implant costs.

- The subacromial balloon spacer has just yet been approved (June 2021) by the FDA and, therefore, the authors have not treated any patients with a subacromial balloon in Boston to date; the experience at the Balgrist is also very limited ( $<10$ cases), with mixed results.

\section{Biomechanics}

The balloon spacer is made of biodegradable copolymer (poly-lactide and $\varepsilon$-caprolactone) that is completely broken down by the body within 1 year. The injected physiological saline solution starts to deflate already after 3 months. The main biomechanical effect propagated is the avoidance or even revision of superior decentration of the humeral head [28].

Another potential indication is protection of an arthroscopic rotator cuff reconstruction using the balloon spacer on top of the repair to reduce the head in the glenohumeral joint during the rotator cuff reconstruction healing period [31].

\section{Results}

The evidence of treatment outcomes is weak, with only mostly level IV and few level III studies showing good short- to mid-term results but no superiority compared to patients treated with APR [22]. In the only level I study by Verma $\mathrm{N}$ et al., no significant differences in clinical outcomes and patient satisfaction were seen between the balloon spacer group $(n=93)$ and the partial repair group $(n=91)$. This study was presented during the ASES annual meeting in October 2020. The study serves as an FDA approval study. Therefore, the study patients were allowed to be treated with a subacromial balloon spacer in the USA. The approval process is still pending, and it remains unclear whether the balloon spacer will be used in the US.

In a systematic review by Stewart RK et al., a total of 10 level IV and 2 level III studies with a mean follow-up of 23 (range 12-52) months were investigated [30]. It was shown that the Constant score was improved between 19 and 50 points by Stewart et al. [30]. The complication rate of $2.1 \%$ was relatively low and included superficial and deep wound infection and balloon spacer dislocation. In 3 of the 12 studies, surgical time was collected, al- beit inconsistently, with implantation time alone lasting an average of $10 \mathrm{~min}$ (range 2-30 min).

A comparison study demonstrated no statistically significant differences in pain and clinical outcomes 1 year postoperatively between APR alone $(n=16)$ and APR and balloon spacer $(n=16)$ patients [22].

\section{Practical conclusion}

- The majority of the authors' younger patients with irreparable posterosuperior tears of the rotator cuff are treated with tendon transfers in combination with partial repair.

- Only in very few selected cases (isolated irreparable superior rupture with pain as the chief complaint) is SCR indicated.

- The subacromial balloon spacer has just been approved in the USA, and in Switzerland the insurance companies are also unwilling to cover the costs. Thus, there is not sufficient experience to be able to make a statement about its use.

- Finally, the evidence of the individual therapy options is mostly based on level IV and a few level III studies, with short- to mid-term follow ups-with the exception of the LTD (10 years follow up). Level I and level II studies with long-term follow-up are necessary to provide clarity on the optimal treatment strategy.

\section{Corresponding address}

\section{Florian Grubhofer, MD}

University Hospital Balgrist, University of Zurich Forchstrasse 340, 8008 Zurich, Switzerland florian.grubhofer@balgrist.ch

Funding. Open access funding provided by University of Zurich

\section{Declarations}

Conflict of interest. J.J.P. Warner declares the following: Stryker: Royalty and consulting on shoulder implants products for joint replacement; Orthospace: Stock for company which produces the Inspace Ballon (no consulting or royalty; this company has been sold to Stryker). F.Grubhofer declares that he has no conflict of interest. 
For this article no studies with human participants or animals were performed by any of the authors. All studies cited were in accordance with the ethical standards indicated in each case.

Open Access. This article is licensed under a Creative Commons Attribution 4.0 International License, which permits use, sharing, adaptation, distribution and reproduction in any medium or format, as long as you give appropriate credit to the original author(s) and the source, provide a link to the Creative Commons licence, and indicate if changes were made. The images or other third party material in this article are included in the article's Creative Commons licence, unless indicated otherwise in a credit line to the material. If material is not included in the article's Creative Commons licence and your intended use is not permitted by statutory regulation or exceeds the permitted use, you will need to obtain permission directly from the copyright holder. To view a copy of this licence, visit http://creativecommons.org/licenses/by/4.0/.

\section{References}

1. Baverel LP, Bonnevialle N, Joudet T et al (2021) Short-term outcomes of arthroscopic partial repair vs. latissimus dorsi tendon transfer in patients with massive and partially repairable rotator cuff tears. J Shoulder Elbow Surg 30:282-289

2. Bertelli JA (2009) Lengthening of subscapularis and transfer of the lower trapezius in the correction of recurrent internal rotation contracture following obstetric brachial plexus palsy. J Bone Joint Surg Br 91:943-948

3. Boileau $P$, Baque F, Valerio L et al (2007) Isolated arthroscopic biceps tenotomy or tenodesis improves symptoms in patients with massive irreparable rotator cuff tears. J Bone Joint Surg Am 89:747-757

4. Burkhart SS, Hartzler RU (2019) Superior capsular reconstruction reverses profound pseudoparalysis in patients with irreparable rotator cuff tears and minimal ornoglenohumeral arthritis. Arthroscopy 35:22-28

5. Burkhart SS, Nottage WM, Ogilvie-Harris DJ et al (1994) Partial repair of irreparable rotator cufftears. Arthroscopy 10:363-370

6. Burkhart SS, Pranckun JJ, Hartzler RU (2020) Superior capsular reconstruction for the operatively irreparable rotator cuff tear: clinical outcomes are maintained 2 years after surgery. Arthroscopy 36:373-380

7. Collin P, Matsumura N, Ladermann A et al (2014) Relationship between massive chronic rotator cuff tear pattern and loss of active shoulder range of motion. JShoulder Elbow Surg 23:1195-1202

8. Cuff DJ, Pupello DR, Santoni BG (2016) Partial rotator cuff repair and biceps tenotomy for the treatment of patients with massive cuff tears and retained overhead elevation: midterm outcomes with a minimum 5 years of follow-up. J Shoulder Elbow Surg 25:1803-1809

9. Cutbush K, Peter NA, Hirpara K (2016) Allarthroscopic latissimus dorsi transfer. Arthrosc Tech 5:e607-e613

10. El-Azab HM, Rott O, Irlenbusch U (2015) Longterm follow-up after latissimus dorsi transfer for irreparable posterosuperior rotator cuff tears. J Bone Joint Surg Am 97:462-469

11. Elhassan B, Bishop A, Shin A et al (2010) Shoulder tendon transfer options for adult patients with brachial plexus injury. J Hand Surg Am 35:1211-1219

\section{Gelenkerhaltende chirurgische Therapieoptionen der posterosuperioren Rotatorenmanschettenruptur. Teilrekonstruktion, SCR, Latissimus Dorsi oder Inferiorer Trapezius Transfer oder Ballon?}

Die Behandlung der irreparablen posterosuperioren Rotatorenmanschettenruptur bei jungen, aktiven Patienten stellt eine Herausforderung dar. Es bestehen verschiedene Therapieoptionen. Nur vereinzelte gelenkserhaltende Therapieoptionen zeigen über einen langen Nachkontrollzeitraum verlässliche Verbesserungen der Schulterfunktion und Patientenzufriedenheit. Die Behandlungsergebnisse der gelenkserhaltenden Operationen sind jedoch nicht mit jenen der RTSA vergleichbar, da die Patienten typischerweise jünger sind und höhere Ansprüche haben.

Bemerkenswert ist, dass zu den meisten gelenkerhaltenden Eingriffen bei irreparablen Rotatorenmanschettenrupturen Langzeittherapieergebnisse fehlen. In dieser Übersichtsarbeit werden die am häufigsten vorgenommenen gelenkerhaltenden Eingriffe hinsichtlich Indikationen, technischer Aspekte und Behandlungsresultaten beleuchtet.

\section{Schlüsselwörter}

Biomechanische Phänomene - Schulterendoprothetik - Sehnentransfer · Schulterprothese . Tenodese

12. Elhassan B, Bishop AT, Hartzler RU et al (2012) Tendon transfer options about the shoulder in patients with brachial plexus injury. J Bone Joint Surg Am 94:1391-1398

13. Elhassan BT, Wagner ER, Werthel JD (2016) Outcome of lower trapezius transfer to reconstruct massive irreparable posterior-superior rotator cuff tear. J Shoulder Elbow Surg 25:1346-1353

14. Elhassan BT, Sanchez-Sotelo J, Wagner ER (2020) Outcome of arthroscopically assisted lower trapezius transfer to reconstruct massive irreparable posterior-superior rotator cuff tears. J Shoulder Elbow Surg 29(10):2135-2142

15. Gerber C (1992) Latissimus dorsi transfer for the treatment of irreparable tears of the rotator cuff. Clin Orthop Relat Res 275:152-160

16. Gerber C, Rahm SA, Catanzaro S et al (2013) Latissimus dorsi tendon transfer for treatment of irreparable posterosuperior rotator cuff tears: long-term results at a minimum follow-up of ten years. JBone Joint Surg Am 95:1920-1926

17. Gerber C, Vinh TS, Hertel R et al (1988) Latissimus dorsi transfer for the treatment of massive tears of the rotator cuff. A preliminary report. Clin Orthop Relat Res 232:51-61

18. Gervasi E, Causero A, Parodi PC et al (2007) Arthroscopic latissimus dorsi transfer. Arthroscopy 23(11):1243.e1-1243.e4

19. Greiner S, KaeaebM, Voss A etal (2021) Comparison of superior capsular reconstruction and partial infraspinatus repair: a matched-pair analysis of irreparable rotator cuff tears. Orthop J Sports Med 9:2325967120984264

20. lagulli ND, Field LD, Hobgood ER et al (2012) Comparison of partial versus complete arthroscopic repair of massive rotator cuff tears. Am J Sports Med 40:1022-1026

21. Jeong JY, Kim SJ, Yoon TH et al (2020) Arthroscopic repair of large and massive rotator cuff tears: complete repair with aggressive release compared with partial repair alone at a minimum follow-up of 5 years. JBone Joint Surg Am 102:1248-1254

22. Malahias MA, Brilakis E, Avramidis G et al (2020) Arthroscopic partial repair with versus without biodegradable subacromial spacer for patients with massive rotator cuff tears: a case-control study. Musculoskelet Surg. https://doi.org/10. 1007/s12306-020-00649-9

23. Malahias MA, Kostretzis L, Chronopoulos E et a (2019) Arthroscopic partial repair for massive rotator cuff tears: Does it work? A systematic review. Sports Med Open 5:13

24. Mihata T, Lee TQ, Hasegawa A et al (2020) Arthroscopic superior capsule reconstruction for irreparable rotator cufftears: comparison of clinical outcomes with and without subscapularis tear. Am JSports Med 48:3429-3438

25. Mihata T, McGarry MH, Pirolo JM et al (2012) Superior capsule reconstruction to restore superior stability in irreparable rotator cuff tears: a biomechanical cadaveric study. Am J Sports Med 40:2248-2255

26. Nimura A, Kato A, Yamaguchi $K$ et al (2012) The superior capsule of the shoulder joint complements the insertion of the rotator cuff. JShoulder Elbow Surg 21:867-872

27. Porcellini G, Castagna A, Cesari Eet al (2011) Partial repair of irreparable supraspinatus tendon tears: clinical and radiographic evaluations at long-term follow-up. JShoulder Elbow Surg 20:1170-1177

28. Savarese E, Romeo R (2012) New solution for massive, irreparable rotator cuff tears: the subacromial "biodegradable spacer". Arthrosc Tech 1:e69-e74

29. Shon MS, Koh KH, Lim TK et al (2015) Arthroscopic partial repair of irreparable rotator cuff tears: preoperative factors associated with outcome deterioration over 2 years. Am J Sports Med 43:1965-1975

30. Stewart RK, Kaplin L, Parada SA et al (2019) Outcomes of subacromial balloon spacer implantation for massive and irreparable rotator cuff tears: a systematic review. Orthop J Sports Med 7:2325967119875717

31. Szollosy G, Rosso C, Fogerty S et al (2014) Subacromial spacer placement for protection of rotator cuff repair. Arthrosc Tech 3:e605-e609

32. Waltenspul M, Jochum B, Filli L et al (2021) Midterm results of arthroscopically-assisted latissimus dorsi transfer for irreparable posterosuperior 


\section{Review Article}

rotator cuff tears. J Shoulder Elbow Surg. https:// doi.org/10.1016/j.jse.2021.03.149

33. Wellmann M, Lichtenberg S, Da Silva G et al (2013) Results of arthroscopic partial repair of large retracted rotator cuff tears. Arthroscopy 29:1275-1282

34. Woodmass JM, Wagner ER, Borque KA et al (2019) Superior capsule reconstruction using dermal allograft: early outcomes and survival. J Shoulder Elbow Surg 28:S100-S109 